\title{
New insights into the association between non-alcoholic fatty liver disease and atherosclerosis
}

\author{
Alejandro Valencia-Rodríguez ${ }^{1}$, Alfonso Vera-Barajas ${ }^{1}$, Beatriz Barranco-Fragoso ${ }^{2}$, Deyanira Kúsulas-Delint ${ }^{1}$, \\ Xingshun $\mathrm{Qi}^{3}$, Nahum Méndez-Sánchez ${ }^{1,4}$ \\ ${ }^{1}$ Liver Research Unit, Medica Sur Clinic \& Foundation, Mexico City, Mexico; ${ }^{2}$ Department of Gastroenterology, National Medical Center “20 \\ Noviembre", Mexico City, Mexico; ${ }^{3}$ General Hospital of Northern Theater Command, Department of Gastroenterology. Shenyang, Liaoning 10016 \\ China; ${ }^{4}$ Faculty of Medicine. National Autonomous University of Mexico, Mexico City, Mexico \\ Correspondence to: Prof. Nahum Méndez-Sánchez, MD, MSc, PhD. Liver Research Unit, Medica Sur Clinic and Foundation, Puente de Piedra 150, \\ Col. Toriello Guerra, ZP. 14050, Mexico City, Mexico. Email: nmendez@medicasur.org.mx; nah@unam.mx. \\ Provenance: This is an invited article commissioned by the Editorial Office, Annals of Translational Medicine. \\ Comment on: Koo BK, Allison MA, Criqui MH, et al. The association between liver fat and systemic calcified atherosclerosis. J Vasc Surg 2019. [Epub \\ ahead of print].
}

Submitted Oct 20, 2018. Accepted for publication Oct 30, 2019.

doi: $10.21037 /$ atm.2019.11.13

View this article at: http://dx.doi.org/10.21037/atm.2019.11.13

In a recent study, Koo et al. (1) showed the association between non-alcoholic fatty liver disease (NAFLD) and systemic calcified atherosclerosis. Those investigators studied 4,185 patients from a community-based cohort evaluated for calcified atherosclerosis in eight different vascular beds, NAFLD was independently associated with calcification in the thoracic aorta and celiac trunk and also with multiarterial calcification (four or more evaluated arteries), even after adjustment for confounders.

There are substantial epidemiological studies that have linked NAFLD with subclinical atherosclerosis by associating it with certain parameters such as flow-mediated vasodilatation. Such an association was inferred from the finding that when a high-resolution external ultrasound is performed to measure brachial artery diameter in the basal state and in response to an increase in blood flow (induced by inflation to $250 \mathrm{mmHg}$ and the deflation of a sphygmomanometer cuff around the forearm), patients with NAFLD show impaired endothelium-dependent flow-mediated vasodilatation (2). Other markers such as an increased carotid artery intima-media thickness obtained by using B-mode carotid ultrasound (3) and arterial stiffness determined by measuring the carotid-femoral pulse wave velocity (PWV) are also associated with NAFLD (4).

Interestingly, in the study from Koo et al. (1) the presence and extent of coronary atherosclerosis coronary artery calcium score was determined by computed tomography. A method previously validated tool to assess coronary heart disease risk. That is important because the analysis has shown that segments with greater calcification tend to have a larger burden of atherosclerotic plaque and percentage lumen stenosis.

On the other hand, many patients included in those studies already had cardiovascular risk factors as well as components of the metabolic syndrome, thereby making it difficult to determine independent associations presenting numerous bias. In Table 1, we showed a summary of systematic reviews and meta-analyses which shows a significant association between NAFLD and CVD. The studies included in those meta-analyses were mainly observational ones, leaving a possibility of debate and even generating disagreement in some of them $(5,6)$. Despite this, in daily practice, it has been suggested that patients with an incidental diagnosis of fatty liver should have a full evaluation of risk factors related to CVD and fibrosis for a better approach and treatment plan with the objective of preventing liver, cardiovascular, and metabolic complications (7). In the next paragraphs, we want to show why patients with NAFLD are prone to develop CVD and the molecular mechanisms involved in this process. 


\section{Impact of NAFLD on the pathogenesis of atherosclerosis}

NAFLD is a clinical entity which should be considered as a multisystem disease closely related to other metabolic diseases, such as diabetes and metabolic syndrome. Fatal as well as non-fatal CVD remain the primary complications in such patients. Although it is difficult to determine the independent relationship of NAFLD with CVD, it is clear that the mechanisms involved in its pathogenesis are extremely complex. It is well known that patients with fatty liver present an increase in visceral adipose tissue (AT) secondary to an inadequate intake of food. This causes a dysfunction in the AT generating insulin resistance (IR), thereby increasing lipolysis and resulting in a greater release of free fatty acids (FFAs) into the bloodstream (8). Additionally, several pro-inflammatory signaling pathways are activated in the AT through two intracellular transcription factors: the nuclear factor $\kappa \beta(\mathrm{NF}-\kappa \beta)$ and the c-Jun N-terminal kinase (JNK). FFAs cause IR in other tissues such as skeletal muscle. This initiates a systemic hyperinsulinemia that reaches the liver and other organs such as the pancreas and heart and promotes the formation of fatty deposits by inducing lipogenesis by the sterol regulatory element-binding protein 1c inhibiting $\beta$-oxidation and establishing steatosis. In the liver, the NF$\mathrm{k} \beta$ pathway is also activated producing pro-inflammatory cytokines such as IL-6, CPR, monocyte-1 chemotactic proteins and $\mathrm{TNF}-\alpha$, consequently amplifying systemic inflammation. Pro-coagulant factors are also increased, such as plasminogen activator inhibitor-1 (PAI-1), fibrinogen and factor VII. Further, there is a greater formation of stress molecules such as reactive oxygen species (ROS) and ceramides, besides a decrease in adiponectin levels, which favor the progression of NAFLD and increases cardiovascular risk (9). All these alterations cause endothelial dysfunction and arterial stiffness, thereby favoring the development of atherosclerosis. In a clinical trial we conducted in 2013, we attempted to explain how the liver is an essential part in the pathogenesis of atherosclerosis, by trying to compensate the endothelial dysfunction with an increase of early endothelial progenitor cells (EPCs). This was sustained by discovering that NAFLD patients had a greater number of these types of cells directly proportional to the degree of steatosis (10). Werner et al. (11) also showed that elevated levels of EPCs were related to a lower risk of CVD. However, in advanced stages of NAFLD in which a cardiovascular disorder may coexist, levels of EPCs are lower (12). Consequently, it would be important to investigate at which point in the progression of NAFLD this protective mechanism is downregulated and therefore favors the development of atherosclerosis. In another study, we also found that subjects who had gallstone disease (GD) exhibited greater carotid atherosclerosis and therefore, an increased cardiovascular risk (13). This reaffirms that metabolism-related diseases are intimately connected to each other by countless pathophysiological mechanisms and for this reason they must be seen as a dynamic process.

\section{Atherosclerotic plaque development}

For the development of atherosclerotic plaque, high concentrations of LDL-C, especially oxidized LDL-C (oxLDL), are almost always necessary (14). These ox-LDL bind with great affinity to macrophage scavenger receptors in the vascular endothelium resulting in the formation of foam cells (Figure 1). Moreover, they induce inflammation by the formation of neo-epitopes that stimulate both humoral and adaptive immunity (14). Therefore, the interaction of metabolic disturbances and the immune system has been increasingly studied, establishing new mechanisms in which the liver can be part. In recent years, the NLRP3 inflammasome has been discovered as an important and key regulator of metabolic inflammation and endothelial dysfunction through the activation of $\mathrm{NF}-\kappa \beta$ pathway. Finally, epigenetic factors are yet another topic of great interest and debate. The microRNA is a single-stranded RNA that acts as a transcription factor for various genes to regulate metabolism (15). Several microRNAs have been implicated both in NAFLD and atherosclerosis. However, the most important to highlight would be microRNA-155 acting as a promoter in cholesterol uptake by expressing scavenger receptors, LOX-1, CD36 and CD68. The other one would be microRNA $33 \mathrm{a} / \mathrm{b}$ which decreases the efflux of cholesterol in macrophages by inhibiting the expression of ABCA1 and NPC1 transporters (15). Another important epigenetic mechanism is advanced glycation end products (AGEs) involved in atherosclerosis development by impairing the anti-atherogenic property of apolipoprotein A-I, resultantly making HDL-C dysfunctional. Furthermore, AGEs inhibit both ABCA1dependent and SR-B-I-mediated cholesterol efflux and thus, reduces reverse cholesterol transport (RCT) (16).

In this context, the study of Koo et al. (1) continues demonstrating an important relationship between NAFLD and atherosclerosis. One of the strengths of this study is 


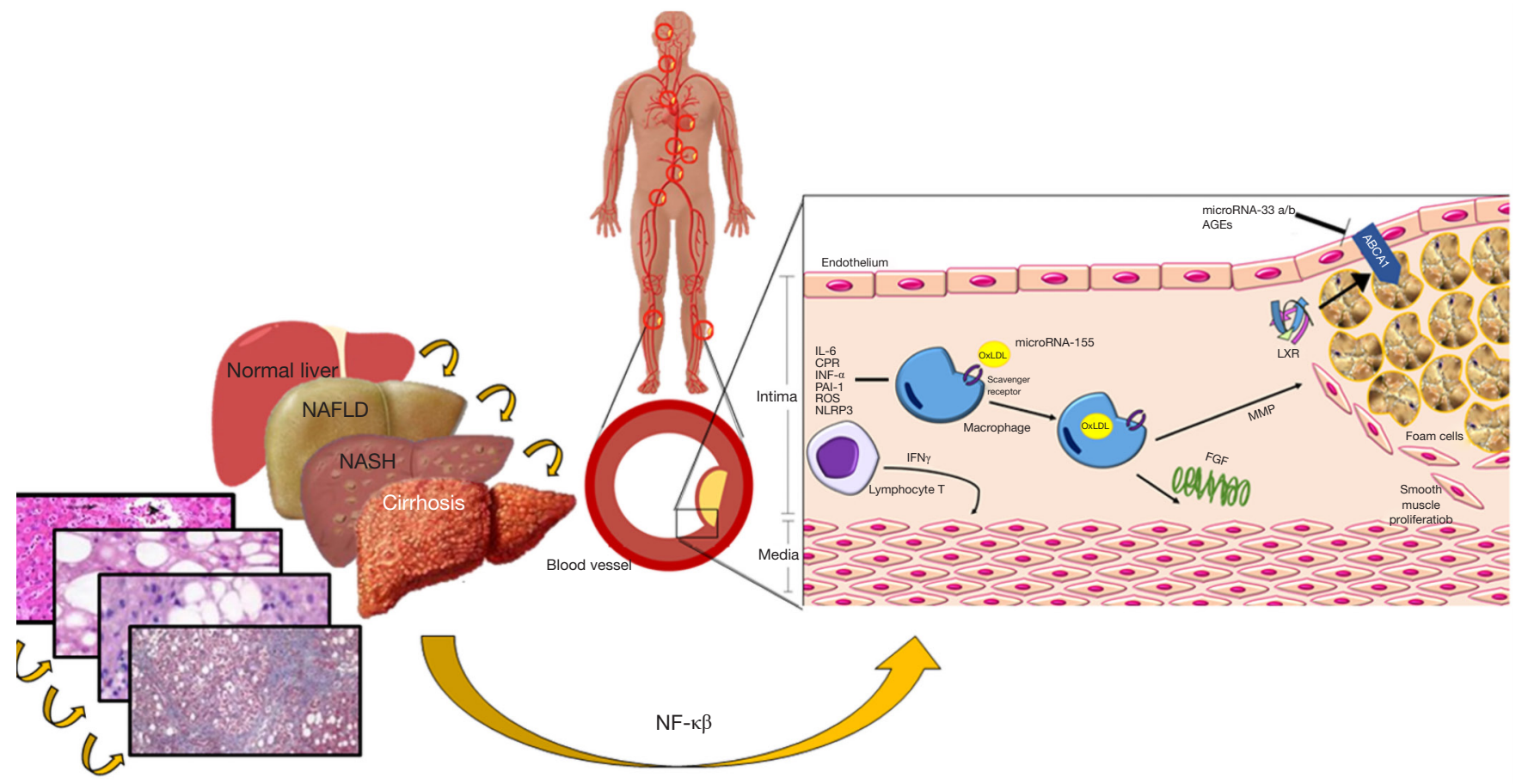

Figure 1 Potential mechanism involved in the development of atherosclerosis. Atherosclerosis is a systemic disease that may involve multiple vessels, according to the stage of fibrosis and inflammation in the liver, there is a greater degree of progression of atherosclerosis. The transcriptional factor NF- $\kappa \beta$ is the main responsible for generating proinflammatory cytokines, procoagulant and oxidative agents such as IL-6, CPR, TNF- $\alpha$, PAI-1, ROS and the NLRP3 inflammasome that favors local recruitment of monocytes and, in smaller numbers, T lymphocytes into the endothelium in conjunction with oxLDL. In the intima, monocytes differentiate into macrophages and internalize oxLDL through scavenger receptors boosted by microRNA-155, subsequently transforming into foam cells. In turn, FGF are produced inducing smooth muscle proliferation forming a fibrous layer in the media layer. On the other hand, T lymphocytes arrive intima producing mediators such as IFN $\gamma$, which affects the ability of smooth muscle cells to produce interstitial collagen, causing instability in the layer. Furthermore, different pro-inflammatory cytokines inhibit the expression of different cholesterol transporters, mainly ABCA1 in foam cells by decreasing their cholesterol flow like microRNA-33 a/b and AGEs. NF- $\kappa \beta$, nuclear factor- $\kappa \beta$; IL-6, interleukin-6; CRP, C-reactive protein; TNF- $\alpha$, tumor necrosis factor alpha; PAI-1, Plasminogen activator inhibitor-1; ROS, Reactive Oxygen Species; NLRP3, NODlike receptor family, pyrin domain containing 3; oxLDL, oxidized low density lipoprotein; FGF, fibroblast growth factor; IFN $\gamma$, interferon gamma; ABCA1, ATP-binding cassette transporter A1; AGEs, advanced glycation end products; MMP, metalloproteinase; LXR, liver X receptor.

that investigators based on calcifications in eight arterial beds. Also, the heterogeneous association between NAFLD and systemic atherosclerosis found in this study has not previously been reported. Conversely, the main shortcoming that was pointed out in this work was its failure of demonstrating an independent association between NAFLD and coronary artery calcification (CAC), however they mentioned something extremely important, their population was mainly non-Hispanic white. Studies that have shown an association between NAFLD and CAC have been in the African-American and Asian population $(17,18)$.
In a meta-analysis carried out where studies from Asia and North America were included, this independent relationship persisted (19), thus racial component might be a predictor in the development of calcification in a specific vascular bed. The other weakness of the study that should be mention is the method they used to diagnose NAFLD, having used CT evaluation many cases of mild hepatic steatosis may have been underdiagnosed and therefore affect the final results. It would also have been interesting to see if more advanced degrees of inflammation and liver fibrosis were related to greater calcification in different vascular beds. 
Table 1 The results of two meta-analyses performed on the association between NAFLD and CVD mortality risk

\begin{tabular}{|c|c|c|c|c|}
\hline Meta-analyzes & Type of studies & Number of participants & Findings & $\mathrm{HR}(95 \% \mathrm{Cl})$ \\
\hline $\begin{array}{l}\text { adverse cardiovascular } \\
\text { events: a systematic } \\
\text { review and }\end{array}$ & & & $\begin{array}{l}\text { NAFLD was associated with an } \\
\text { increased risk of prevalent CVD }\end{array}$ & $1.81(1.23-2.66)$ \\
\hline $\begin{array}{l}\text { Nonalcoholic fatty } \\
\text { liver disease and } \\
\text { risk of incident } \\
\text { cardiovascular disease: }\end{array}$ & $\begin{array}{l}6 \text { community-based cohort } \\
5 \text { outpatient cohort } \\
2 \text { hospital-based-cohort }\end{array}$ & $\begin{array}{l}34,043,46 \% \text { men, } \\
\text { mainly from Asia and } \\
\text { USA }\end{array}$ & $\begin{array}{l}\text { Patients with NAFLD had a } \\
\text { higher risk of fatal and/or non- } \\
\text { fatal CVD events than those } \\
\text { without NAFLD }\end{array}$ & $1.64(1.26-2.13)$ \\
\hline
\end{tabular}

NAFLD, nonalcoholic fatty liver disease; CVD, cardiovascular disease.

In conclusion, NAFLD is closely related to CVD through different metabolic and immune mechanisms that underlie the disease. However, more clinical and experimental studies are required to explain this relationship between NAFLD and CVD in more detail.

\section{Acknowledgments}

This study was partly supported by a grant from Medica Sur Clinic and Foundation.

\section{Footnote}

Conflicts of Interest: The authors have no conflicts of interest to declare.

Ethical Statement: The authors are accountable for all aspects of the work in ensuring that questions related to the accuracy or integrity of any part of the work are appropriately investigated and resolved.

\section{References}

1. Koo BK, Allison MA, Criqui MH, et al. The association between liver fat and systemic calcified atherosclerosis. J Vasc Surg 2019. [Epub ahead of print].

2. Villanova N, Moscatiello S, Ramilli S, et al. Endothelial dysfunction and cardiovascular risk profile in nonalcoholic fatty liver disease. Hepatology 2005;42:473-80.
3. Brea A, Mosquera D, Martín E, et al. Nonalcoholic fatty liver disease is associated with carotid atherosclerosis: a case-control study. Arterioscler Thromb Vasc Biol 2005;25:1045-50.

4. Salvi P, Ruffini R, Agnoletti D, et al. Increased arterial stiffness in nonalcoholic fatty liver disease: The CardioGOOSE study. J Hypertens 2010;28:1699-707.

5. Wu S, Wu F, Ding Y, et al. Association of non-alcoholic fatty liver disease with major adverse cardiovascular events: A systematic review and meta-analysis. Sci Rep 2016;6:33386.

6. Targher G, Byrne CD, Lonardo A, et al. Non-alcoholic fatty liver disease and risk of incident cardiovascular disease: A meta-analysis. J Hepatol 2016;65:589-600.

7. Méndez-Sánchez N, Chavez-Tapia NC, Almeda-Valdes $\mathrm{P}$, et al. The management of incidental fatty liver found on imaging. What do we need to do? Am J Gastroenterol 2018;113:1274-6.

8. Mendez-Sanchez N, Cruz-Ramon VC, Ramirez-Perez OL, et al. New Aspects of Lipotoxicity in Nonalcoholic Steatohepatitis. Int J Mol Sci 2018;19. doi: 10.3390/ ijms19072034.

9. Targher G, Day CP, Bonora E. Risk of cardiovascular disease in patients with nonalcoholic fatty diver Disease. $\mathrm{N}$ Engl J Med 2010;363:1341-50.

10. Gutiérrez-Grobe Y, Gavilanes-Espinar JG, Masso-Rojas FA, et al. Metabolic syndrome and nonalcoholic fatty liver disease. The role of endothelial progenitor cells. Ann Hepatol 2013;12:908-14. 
11. Werner N, Kosiol S, Schiegl T, et al. Circulating endothelial progenitor cells and cardiovascular outcomes. N Engl J Med 2005;353:999-1007.

12. Chiang CH, Huang PH, Chung FP, et al. Decreased circulating endothelial progenitor cell levels and function in patients with nonalcoholic fatty liver disease. PLoS One 2012;7:e31799.

13. Méndez-Sánchez N, Zamora-Valdés D, Flores-Rangel JA, et al. Gallstones are associated with carotid atherosclerosis. Liver Int 2008;28:402-6.

14. Libby P, Buring JE, Badimon L, et al. Atherosclerosis. Nat Rev Dis Primers 2019;5:56.

15. Shao D, Lian Z, Di Y, et al. Dietary compounds have potential in controlling atherosclerosis by modulating macrophage cholesterol metabolism and inflammation via miRNA. NPJ Sci Food 2018;2:13.

Cite this article as: Valencia-Rodríguez A, Vera-Barajas A, Barranco-Fragoso B, Kúsulas-Delint D, Qi X, Méndez-Sánchez N. New insights into the association between non-alcoholic fatty liver disease and atherosclerosis. Ann Transl Med 2019;7(Suppl 8):S300. doi: 10.21037/atm.2019.11.13
16. Srivastava RAK. Life-style-induced metabolic derangement and epigenetic changes promote diabetes and oxidative stress leading to NASH and atherosclerosis severity. J Diabetes Metab Disord 2018;17:381-91.

17. Liu J, Musani SK, Bidulescu A, et al. Fatty liver, abdominal adipose tissue and atherosclerotic calcification in African Americans: The Jackson Heart Study. Atherosclerosis 2012;224:521-5.

18. Wu R, Hou F, Wang X, et al. Nonalcoholic Fatty Liver Disease and Coronary Artery Calcification in a Northern Chinese Population: a Cross Sectional Study. Sci Rep 2017;7:9933.

19. Jaruvongvanich V, Wirunsawanya K, Sanguankeo A, et al. Nonalcoholic fatty liver disease is associated with coronary artery calcification: A systematic review and meta-analysis. Dig Liver Dis 2016;48:1410-7. 